\title{
Robot Team Teleoperation for Cooperative Manipulation using Wearable Haptics
}

\author{
Selma Musić ${ }^{1}$, Gionata Salvietti ${ }^{2}$, Pablo Budde gen. Dohmann ${ }^{1}$, Francesco Chinello ${ }^{3}$, \\ Domenico Prattichizzo ${ }^{2}$, Sandra Hirche ${ }^{1}$
}

\begin{abstract}
Robot teams require planning and adaptive capabilities in order to perform cooperative manipulation tasks in dynamic or unstructured environments. Since these capabilities are inherent to humans, it is suitable to consider humanrobot team teleoperation for cooperative manipulation where a single human collaborates with the robot team. In this paper, we present a subtask-based control approach which enables a simultaneous execution of two subtasks by the robot team, interacting with the object: trajectory tracking and formation preservation. Control inputs for both subtasks are provided by the human operator. The commands are projected onto the spaces of subtasks using a command mapping strategy. Analogously, measured interacting forces are projected onto the space of feedback signals, provided to the human via wearable fingertip haptic devices through a feedback mapping strategy. Experimental results validate the proposed approach.
\end{abstract}

\section{INTRODUCTION}

The research area of human-robot interaction mostly focuses on the interaction between a single human and a single robot. Reduction of price, size and operational complexity of modern robots, as well as the advances in communication technology are enablers for multi-robot systems which perform tasks in cooperation. The advantages of robot teams compared to single robots are increased flexibility, reliability and robustness. Even though robot teams are capable of performing many functions autonomously with high precision and repeatability, they still need intervention and guidance in the form of high level reasoning and (re)planning. Since these capabilities are inherent to humans, it is reasonable to employ human-robot team interaction.

Human-robot team interaction has been investigated mostly as a form of remote interaction. Typically, shared control approaches are applied, where the human operator conducts high-level tasks while the robots conduct low-level tasks autonomously [1]. The way in which the human interacts with the robot team can be in-the-loop (human actively controls the system on the physical level) or on-the-loop (human supervises and occasionally controls the system on a symbolic level).

Human-in-the-loop control approaches for human-robot team interaction are typically based on the existing bilateral teleoperation control approaches. The human commands the

\footnotetext{
${ }^{1}$ Chair of Information-oriented Control, Technische Universität München, Germany

${ }^{2}$ Department of Information Engineering and Mathematics, University of Siena, Italy

${ }^{3}$ Dept. of Business Development and Technology, Aarhus University, Denmark
}

motion of the robot team, while the robot team coordinates autonomously, see e.g. [2] and [3]. The interaction is achieved via a haptic device. Teleoperation of robot teams in which the human operator is not coupled to a haptic device is investigated in [4] and [5] (hand motion tracked with cameras) and in [6] (commands provided via a tablet). However, the feedback provided to the human in this case is visual, which is insufficient when the robots interact with the environment.

One of the challenges in the human-robot team scenario is the multi-contact interaction, where the robot team establishes multiple contacts with the environment. A classical example of such interaction is bilateral telemanipulation where the human hand is in correspondence with a multifingered robotic hand. For example, in [7], the human teleoperates the robot team manipulating an object, but due to the lack of force feedback, the human commands result in the abrupt pulling of the object. Novel forms of multi-contact interaction between humans and robots within teleoperation scenarios are possible thanks to the availability of wearable haptic devices, e.g. [8]. Furthermore, due to their wearability it is possible to establish teleoperation in which the human moves freely and, therefore, operates in an extended workspace.

An important property of robot teams is their capability to perform multiple subtasks simultaneously due to their inherent redundancy, which can enhance their operation in unstructured environments. In order to avoid conflicts between subtasks, a null-space based behavioral control approach is used for robot swarms [9]. It is inspired by the redundancy resolution control approaches for robot manipulators. However, the control is only kinematic and, therefore, unsuitable for physical interactions in the environment, necessary for cooperative manipulation tasks.

In this paper, a novel human-robot team interaction is introduced where a human teleoperates a robot team to perform a cooperative manipulation task. We propose a behavioralbased control approach which decouples the cooperative manipulation task into subtasks, performed simultaneously. The approach is suitable for tasks in which the robot team interacts with environment. We enhance the interaction by enabling the human operator to move freely in the workspace, which is achieved with wearable thimble haptic devices. Proposed command mapping enables the human to control the robot team on the subtask level, instead of controlling individual robots, and feedback mapping establishes the transparency of the interaction forces. Suitability 


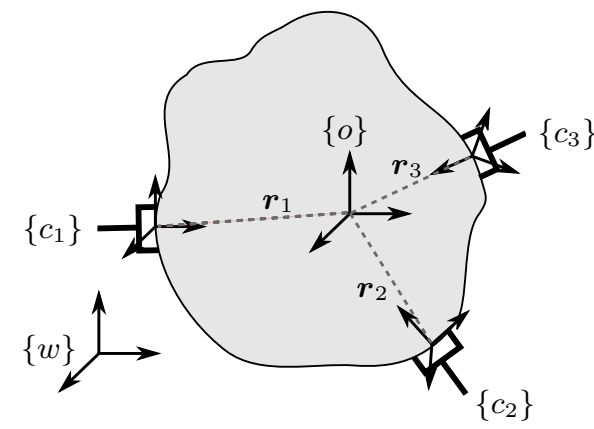

Fig. 1. Robot team with $N$ manipulators grasps and manipulates a common object. Frames $\left\{c_{i}\right\}, i=1, \ldots, N$, are assigned to the end-effectors and frame $\{o\}$ is assigned to the object. Distances between $\left\{c_{i}\right\}$ and $\{o\}$ in $\{w\}$ are $\boldsymbol{r}_{i} i=1, \ldots, N$.

of the approach is experimentally validated, with human, equipped with two wearable thimble devices, commanding grasp maintenance and object manipulation subtasks to the team of robot manipulators.

The paper is organized as follows: in Sec. II a dynamical subtask-based control approach is proposed on an example of the cooperative manipulation task. Sec. III provides mapping approaches that include human in the loop. Experimental results and conclusive remarks are provided in Sec. IV and Sec. V, respectively.

\section{ROBOT TEAM DYNAMICAL MODEL AND CONTROL}

In this section, the dynamical model of the robot team and the control for a cooperative manipulation task is introduced. Let us assume that $N$ robot manipulators form the robot team which cooperatively grasps and manipulates an object, as shown in Fig. 2. Furthermore, let us assume that each manipulator can make only one contact point with the object. The pose of the $i$-th manipulator in the task space is the pose of the frame $\left\{c_{i}\right\}$ in $\{w\}$, given with the vector $\boldsymbol{x}_{i}=$ $\left[\boldsymbol{p}_{i}^{T}, \boldsymbol{q}_{i}^{T}\right]^{T}$, where $\boldsymbol{p}_{i} \in \mathbb{R}^{3}$ is a position vector and $\boldsymbol{q}_{i} \in$ $\operatorname{Spin}(3)$ is a unit quaternion representing the orientation of the end-effector. The pose of the object frame $\{o\}$ in $\{w\}$ is given with the vector $\boldsymbol{x}_{o}=\left[\boldsymbol{p}_{o}^{T}, \boldsymbol{q}_{o}^{T}\right]^{T}$.

The dynamics of a single robot manipulator, $i$, in the task space can be described with Euler-Lagrange equation:

$$
\boldsymbol{M}_{i}\left(\boldsymbol{x}_{i}\right) \dot{\boldsymbol{v}}_{i}+\boldsymbol{c}_{i}\left(\boldsymbol{x}_{i}, \boldsymbol{v}_{i}\right)+\boldsymbol{g}_{i}\left(\boldsymbol{x}_{i}\right)=\boldsymbol{h}_{i}+\boldsymbol{h}_{i}^{m},
$$

where $\boldsymbol{v}_{i}=\left[\dot{\boldsymbol{p}}_{i}^{T}, \boldsymbol{\omega}_{i}^{T}\right]^{T} \in \mathbb{R}^{6}$ is the velocity vector with $\dot{\boldsymbol{p}}_{i}$ and $\boldsymbol{\omega}_{i}$ being translational and angular velocities, respectively. The inertial matrix of the manipulator is $\boldsymbol{M}_{i}\left(\boldsymbol{x}_{i}\right) \in$ $\mathbb{R}^{6 \times 6}, \boldsymbol{c}_{i}\left(\boldsymbol{x}_{i}, \boldsymbol{v}\right) \in \mathbb{R}^{6}$ is the vector of Coriolis and centrifugal forces, $\boldsymbol{g}_{i}\left(\boldsymbol{x}_{i}\right) \in \mathbb{R}^{6}$ is the vector of gravitational forces, $\boldsymbol{h}_{i} \in \mathbb{R}^{6}$ is the vector of control input wrenches and $\boldsymbol{h}_{i}^{m}=$ $\left[\boldsymbol{f}_{i}^{m T}, \boldsymbol{t}_{i}^{m T}\right]^{T} \in \mathbb{R}^{6}$ is the vector of measured wrenches with $\boldsymbol{f}_{i}^{m T}$ and $\boldsymbol{t}_{i}^{m T}$ being force and torque vectors, respectively. The multi-robot dynamical model is obtained by stacking the $N$ dynamical equations (1) for $N$ robots in

$$
\boldsymbol{M}(\boldsymbol{x}) \dot{\boldsymbol{v}}+\boldsymbol{c}(\boldsymbol{x}, \boldsymbol{v})+\boldsymbol{g}(\boldsymbol{x})=\boldsymbol{h}+\boldsymbol{h}^{m},
$$

where $\boldsymbol{M}(\boldsymbol{x})=\operatorname{blockdiag}\left(\boldsymbol{M}_{1}(\boldsymbol{x}), \ldots, \boldsymbol{M}_{i}(\boldsymbol{x}), \ldots, \boldsymbol{M}_{N}(\boldsymbol{x})\right)$ is the inertial matrix of the multi-robot system, $c=$
$\left[\boldsymbol{c}_{1}^{T}, \ldots, \boldsymbol{c}_{i}^{T}, \ldots, \boldsymbol{c}_{N}^{T}\right]^{T}$ is the stacked vector of Coriolis and centrifugal forces and $\boldsymbol{g}=\left[\boldsymbol{g}_{1}^{T}, \ldots, \boldsymbol{g}_{i}^{T}, \ldots, \boldsymbol{g}_{N}^{T}\right]^{T}$ is the stacked vector of gravitational forces. The stacked control input wrenches are $\boldsymbol{h}=\left[\boldsymbol{h}_{1}^{T}, \ldots, \boldsymbol{h}_{i}^{T}, \ldots, \boldsymbol{h}_{N}^{T}\right]^{T}$ and the stacked measured wrenches are $\boldsymbol{h}^{m}=\left[\boldsymbol{h}_{1}^{m T}, \ldots, \boldsymbol{h}_{i}^{m T}, \ldots, \boldsymbol{h}_{N}^{m T}\right]^{T}$. Stacked vectors of pose, velocity and acceleration are $\boldsymbol{x}, \boldsymbol{v}$, and $\dot{v}$, respectively.

\section{A. Dynamical subtask-based control for robot teams}

Dynamical subtask-based control for robot teams is a control approach that defines multiple dynamical subtasks that are performed simultaneously by the robot team. The main idea behind the approach is to project the system states on a lower dimensional space of subtasks. Each subtask defines a team behavior that can be described by a model that evolves on the corresponding subtask space. Additionally, the controller can be implemented on the subtask level as well. The space of subtasks is defined by a proper coordinate transformation

$$
\boldsymbol{x}_{s, j}=\boldsymbol{f}(\boldsymbol{x}) \in \mathbb{S}, \quad j=1, \ldots, r
$$

where $\boldsymbol{x}_{s, j} \in \mathbb{S}$ is the vector of coordinates for the $j^{\text {th }}$ subtask out of $r$ subtasks and $\boldsymbol{f}(\boldsymbol{x})$ is a differentiable coordinate transformation to the subtask manifold $\mathbb{S}$. Velocities of the $j^{\text {th }}$ subtask are defined with

$$
\boldsymbol{v}_{s, j}=\frac{\partial \boldsymbol{f}(\boldsymbol{x})}{\partial \boldsymbol{x}} \boldsymbol{v}=\boldsymbol{J}_{s, j}(\boldsymbol{x}) \boldsymbol{v}, \quad j=1, \ldots, r
$$

where $\boldsymbol{J}_{s, j}(\boldsymbol{x}) \in \mathbb{R}^{n_{j} \times n}$ is the full-rank subtask Jacobian. The solution of Stacking all the subtask definitions (4) into a single matrix equation

$$
\boldsymbol{v}_{s}=\boldsymbol{J}_{s}(\boldsymbol{x}) \boldsymbol{v}
$$

gives a set of constraints applied on the system. In principle, the solution of (5) is not unique and can be computed by minimizing the kinetic energy

$$
\begin{aligned}
& \min _{\boldsymbol{v}} \frac{1}{2} \boldsymbol{v}^{T} \boldsymbol{M}(\boldsymbol{x}) \boldsymbol{v} \\
& \text { s.t. } \boldsymbol{J}_{s}(\boldsymbol{x}) \boldsymbol{v}-\boldsymbol{v}_{s}=0 .
\end{aligned}
$$

The solution is obtained through inertia-weighted pseudoinverse of the subtask Jacobian:

$$
\boldsymbol{J}_{s}^{\# M}=\boldsymbol{M}(\boldsymbol{x})^{-1} \boldsymbol{J}_{s}^{T}\left(\boldsymbol{J}_{s} \boldsymbol{M}(\boldsymbol{x})^{-1} \boldsymbol{J}_{s}^{T}\right)^{-1} .
$$

It is possible to write the solution of $v$ as

$$
\boldsymbol{v}=\boldsymbol{J}_{s, 1}^{\# M} \boldsymbol{v}_{s, 1}+\ldots+\boldsymbol{J}_{s, r}^{\# M} \boldsymbol{v}_{s, r}
$$

if the following holds

$$
\left(\boldsymbol{J}_{s, j}^{\# M}\right)^{T} \boldsymbol{M}(\boldsymbol{x}) \boldsymbol{J}_{s, k}^{\# M}=0, \quad \forall j \neq k .
$$

If the Coriolis and centrifual terms are compensated, the equation (9) implies that the subtasks are decoupled and evolve on distinct subtask manifolds.

Let us now perform dynamic decoupling on the multi-robot system to decouple the multi-robot dynamics (2), and control two subtasks separately. 
For the cooperative manipulation task, robots in the multirobot system need to move as a single, tightly coupled entity. In order to achieve that, we formulate two subtasks as cooperative (object manipulation) and relative (grasp maintenance).

\section{B. Cooperative behavior}

Cooperative behavior of the robot team is suitable to perfom object manipulation in the cooperative manipulation task. Therefore, it is necessary that the robot team moves as a single entity in its task space. This can be performed by choosing a point that represent the overall behavior of the robot team.

Let us define the motion variables for the cooperative subtask with the pose vector $\boldsymbol{x}_{c}=\left[\boldsymbol{p}_{c}^{T}, \boldsymbol{q}_{c}^{T}\right]^{T}$. Furthermore, let us assume that $\boldsymbol{x}_{o}=\boldsymbol{x}_{c}$. The point $\boldsymbol{x}_{c}$ is computed from the poses of the end-effectors in the task space by imposing a constraint on the relative orientation of the object and the end-effectors, i.e. ${ }^{o} \delta \boldsymbol{q}_{i}=\boldsymbol{c}_{i}=$ const. and $\boldsymbol{\omega}_{i}=\boldsymbol{\omega}_{o}$. The transformation is

$$
\boldsymbol{x}_{c}=\left[\begin{array}{c}
\boldsymbol{p}_{c} \\
\boldsymbol{q}_{c}
\end{array}\right]=\boldsymbol{f}_{c}(\boldsymbol{x})=\frac{1}{N}\left[\begin{array}{c}
\sum_{i=1}^{N}\left(\boldsymbol{p}_{i}-\boldsymbol{R}_{o}{ }^{o} \boldsymbol{r}_{i}\right) \\
\frac{\sum_{i=1}^{N} \boldsymbol{q}_{i} \star \boldsymbol{c}_{i}}{\left\|\sum_{i=1}^{N} \boldsymbol{q}_{i} \star \boldsymbol{c}_{i}\right\|}
\end{array}\right],
$$

where $\boldsymbol{R}_{o}$ is the rotational matrix of the frame $\{o\}$ w.r.t. the frame $\{w\}$. or in the form of (4) as

$$
\boldsymbol{v}_{c}=\underbrace{\frac{1}{N}\left[\begin{array}{ccccc}
I_{3} & \boldsymbol{S}\left(\boldsymbol{r}_{1}\right) & \ldots & I_{3} & \boldsymbol{S}\left(\boldsymbol{r}_{N}\right) \\
0_{3} & I_{3} & \ldots & 0_{3} & I_{3}
\end{array}\right]}_{\boldsymbol{J}_{c}} \boldsymbol{v}
$$

where $\boldsymbol{v}_{c}=\left[\dot{\boldsymbol{p}}_{c}^{T}, \boldsymbol{\omega}_{c}^{T}\right]^{T} \in \mathbb{R}^{6}$ is the cooperative velocity vector, $\boldsymbol{J}_{c} \in \mathbb{R}^{6 \times n}$ is the cooperative subtask Jacobian, and $\boldsymbol{S}()$ is a skew-symmetric matrix.

Cooperative subtask Jacobian is related to the grasp matrix from the grasp and cooperative manipulation theory with the equality $\boldsymbol{J}_{c}=\boldsymbol{G}^{\dagger T}$, where $\boldsymbol{G}$ represent the grasp matrix [12]. We can say that the subtask Jacobian projects the variables from a high-dimensional space of the robot team onto a lower-dimensional space of a subtask. This dimensionality reduction eases the control design. The weighted Jacobian for the cooperative task is $\overline{\boldsymbol{J}}_{c}$.

Additionally, the transformation of wrenches is possible with

$$
\boldsymbol{h}_{c}=\left(\boldsymbol{J}_{c}^{\# M}\right)^{T} \boldsymbol{h},
$$

where $\boldsymbol{h}_{c} \in \mathbb{R}^{6}$ is a wrench acting on the cooperative subtask level.

\section{Relative behavior}

The relative subtask is suitable for performing grasping and grasp maintenance. It is described by the relative behavior of the robot team, which defines the shape of its formation in the task space. The relative motion between the robots is only allowed along the normals to the object surface, i.e. along the axis $\hat{\boldsymbol{n}}_{i}$ of the frame $\left\{c_{i}\right\}$. In the context of grasp theory these constraints are hard finger constraints, since the relative rotational motions are excluded. The coordinate transformation for the relative subtask are, therefore, defined as:

$$
\boldsymbol{x}_{r}=\left[\begin{array}{c}
\boldsymbol{p}_{r 1} \\
{ }^{2} \boldsymbol{q}_{r 1} \\
\vdots \\
\boldsymbol{p}_{r(N-1)} \\
{ }^{N} \boldsymbol{q}_{r(N-1)}
\end{array}\right]=\left[\begin{array}{c}
\boldsymbol{p}_{1}-\boldsymbol{p}_{2}-\boldsymbol{R}_{o}{ }^{o} \boldsymbol{r}_{12} \\
\boldsymbol{q}_{2}^{-1} \star \boldsymbol{q}_{1} \\
\vdots \\
\boldsymbol{p}_{(N-1)}-\boldsymbol{p}_{N}-\boldsymbol{R}_{o}{ }^{o} \boldsymbol{r}_{(N-1) N} \\
\boldsymbol{q}_{N}^{-1} \star \boldsymbol{q}_{N-1}
\end{array}\right]
$$

or in the form of (4) as:

$$
\boldsymbol{v}_{r}=\underbrace{\left[\begin{array}{ccccccc}
\boldsymbol{I}_{3} & \boldsymbol{S}\left(\boldsymbol{r}_{12}\right) & -\boldsymbol{I}_{3} & \mathbf{0}_{3} & \cdots & \mathbf{0}_{3} & \mathbf{0}_{3} \\
\mathbf{0}_{3} & \boldsymbol{I}_{3} & \mathbf{0}_{3} & -\boldsymbol{I}_{3} & \cdots & \mathbf{0}_{3} & \mathbf{0}_{3} \\
\vdots & \vdots & \vdots & \vdots & \ddots & \vdots & \vdots \\
\mathbf{0}_{3} & \mathbf{0}_{3} & \mathbf{0}_{3} & \mathbf{0}_{3} & \cdots & -\boldsymbol{I}_{3} & \mathbf{0}_{3} \\
\mathbf{0}_{3} & \mathbf{0}_{3} & \mathbf{0}_{3} & \mathbf{0}_{3} & \cdots & \mathbf{0}_{3} & -\boldsymbol{I}_{3}
\end{array}\right]}_{\boldsymbol{J}_{r}} \boldsymbol{v}
$$

where $\boldsymbol{J}_{r} \in \mathbb{R}^{(n-6) \times n}$ is the full-rank relative subtask Jacobian. It is possible to show that the condition (9) is satisfied in this case:

$$
\left(\boldsymbol{J}_{c}^{\# M}\right)^{T} \boldsymbol{M}(\boldsymbol{x}) \boldsymbol{J}_{r}^{\# M}=0 .
$$

We conclude that the space of the relative subtask $\mathbb{S}_{r}$ is orthogonal to the space of the cooperative subtask $\mathbb{S}_{c}$. Transformation of wrenches is possible with the following equation:

$$
\boldsymbol{h}_{r}=\left(\boldsymbol{J}_{r}^{\# M}\right)^{T} \boldsymbol{h}
$$

\section{Dynamic decoupling and control}

Let us compute the acceleration of the robot team by derivating (4)

$$
\dot{\boldsymbol{v}}=\boldsymbol{J}_{s}^{\# M} \dot{\boldsymbol{v}}_{s}+\dot{\boldsymbol{J}}_{s}^{\# M} \boldsymbol{v}_{s}
$$

where $\boldsymbol{J}_{s}=\left[\boldsymbol{J}_{c}^{T} \boldsymbol{J}_{r}^{T}\right]^{T}$ and $\boldsymbol{v}_{s}=\left[\boldsymbol{v}_{c}^{T} \boldsymbol{v}_{r}^{T}\right]^{T}$, and insert it into (2. Furthermore, let us multiply the same equation with $\left(\boldsymbol{J}_{s}^{\# M}\right)^{T}$. Since (15) holds, it is possible to write the dynamical equation for cooperative and relative subtask. More specifically, for the relative subtask, following holds:

$$
\begin{aligned}
& \left(\boldsymbol{J}_{c}^{\# M}\right)^{T}\left[\boldsymbol{M}(\boldsymbol{x}) \boldsymbol{J}_{c}^{\# M} \dot{\boldsymbol{v}}_{c}+\boldsymbol{M}(\boldsymbol{x}) \dot{\boldsymbol{J}}_{c}^{\# M} \boldsymbol{v}_{c}+\right. \\
& \boldsymbol{c}(\boldsymbol{x}, \boldsymbol{v})+\boldsymbol{g}(\boldsymbol{x})]=\left(\boldsymbol{J}_{c}^{\# M}\right)^{T}\left(\boldsymbol{h}+\boldsymbol{h}^{m}\right),
\end{aligned}
$$

which, using (12), can be rewritten as:

$$
\boldsymbol{M}_{c}(\boldsymbol{x}) \dot{\boldsymbol{v}}_{c}+\boldsymbol{c}_{c}(\boldsymbol{x}, \boldsymbol{v})+\boldsymbol{g}_{c}(\boldsymbol{x})=\boldsymbol{h}_{c}+\boldsymbol{h}_{c}^{m},
$$

where $\boldsymbol{h}_{c}^{m}=\left[\boldsymbol{f}_{c}^{m T}, \boldsymbol{t}_{c}^{m T}\right]^{T}$ represents external measured wrenches. We propose the Cartesian impedance controller for achieving a desired cooperative behavior:

$$
\begin{aligned}
& \boldsymbol{h}_{c}=\boldsymbol{c}_{c}(\boldsymbol{x}, \boldsymbol{v})+\boldsymbol{g}_{c}(\boldsymbol{x})+ \\
& \boldsymbol{M}_{c}(\boldsymbol{x}) \dot{\boldsymbol{v}}_{c}^{d}+\boldsymbol{D}_{c}\left(\boldsymbol{v}_{c}-\boldsymbol{v}_{c}^{d}\right)+\boldsymbol{h}_{c}^{K}\left(\boldsymbol{x}_{c}, \boldsymbol{x}_{c}^{d}\right)
\end{aligned}
$$

where $\boldsymbol{D}_{c}=$ blockdiag $\left[d_{c, t} \boldsymbol{I}_{3}, d_{c, r} \boldsymbol{I}_{3}\right]$ is the damping matrix of the controller and the geometrically consistent stiffness $\boldsymbol{h}_{c}^{K}[13]$ is given as

$$
\boldsymbol{h}_{c}^{K}=\left[\begin{array}{c}
k_{c} I_{3} \Delta \boldsymbol{p}_{c} \\
\kappa_{c}^{\prime} I_{3} \Delta \boldsymbol{\epsilon}_{c}
\end{array}\right]
$$


where $\Delta \boldsymbol{p}_{c}=\boldsymbol{p}_{c}-\boldsymbol{p}_{c}^{d}$ is the difference between actual and desired position. Vector $\Delta \epsilon_{c}$ is a part of the relative orientation vector $\Delta \boldsymbol{q}_{c}=\left(\Delta \eta_{c}, \Delta \boldsymbol{\epsilon}_{c}^{T}\right)^{T}$, where the relative orientation is $\Delta \boldsymbol{q}_{c}=\boldsymbol{q}_{c} *\left(\boldsymbol{q}_{c}^{d}\right)^{-1}$. The rotational stiffness parameter is given as $\kappa_{c}=2 \Delta \eta_{c} \kappa_{c}$.

Analogously, relative subtask dynamics can be rewritten as:

$$
\begin{aligned}
& \left(\boldsymbol{J}_{r}^{\# M}\right)^{T}\left[\boldsymbol{M}(\boldsymbol{x}) \boldsymbol{J}_{r}^{\# M} \dot{\boldsymbol{v}}_{r}+\boldsymbol{M}(\boldsymbol{x}) \dot{\boldsymbol{j}}_{r}^{\# M} \boldsymbol{v}_{r}+\right. \\
& \boldsymbol{c}(\boldsymbol{x}, \boldsymbol{v})+\boldsymbol{g}(\boldsymbol{x})]=\left(\boldsymbol{J}_{r}^{\# M}\right)^{T}\left(\boldsymbol{h}+\boldsymbol{h}^{m}\right),
\end{aligned}
$$

which, using (16), can be rewritten as:

$$
\boldsymbol{M}_{r}(\boldsymbol{x}) \dot{\boldsymbol{v}}_{r}+\boldsymbol{c}_{r}\left(\boldsymbol{x}, \boldsymbol{v}_{r}\right)+\boldsymbol{g}_{r}(\boldsymbol{x})=\boldsymbol{h}_{r}+\boldsymbol{h}_{r}^{m}
$$

where $\boldsymbol{h}_{r}^{m}=\left[\boldsymbol{f}_{r}^{m}, \boldsymbol{t}_{r}^{m}\right]^{T}$ represents measured internal wrenches. For the relative dynamics we propose Cartesian compliance controller, suitable for safe and stable interaction with the object:

$$
\begin{aligned}
& \boldsymbol{h}_{r}=\boldsymbol{c}_{r}\left(\boldsymbol{x}, \boldsymbol{v}_{r}\right)+\boldsymbol{g}_{r}(\boldsymbol{x})+ \\
& \boldsymbol{D}_{r}\left(\boldsymbol{v}_{r}-\boldsymbol{v}_{r}^{d}\right)+\boldsymbol{h}_{r}^{K}\left(\boldsymbol{x}_{r}, \boldsymbol{x}_{r}^{d}\right)
\end{aligned}
$$

where $\boldsymbol{D}_{r}=$ blockdiag $\left[d_{r, t} \boldsymbol{I}_{3}, d_{r, r} \boldsymbol{I}_{3}\right]$ is the damping matrix and the geometrically consistent stiffness $\boldsymbol{h}_{r}^{K}$ is

$$
\boldsymbol{h}_{r}^{K}=\left[\begin{array}{c}
k_{r} I_{3} \Delta \boldsymbol{p}_{r} \\
\kappa_{r}^{\prime} R_{i} \Delta^{i} \boldsymbol{\epsilon}_{r}
\end{array}\right] .
$$

With (19) and (23) and controllers (20) and (24), we obtain geometrically decoupled dynamics of a cooperative manipulation system. It is possible to show conditional stability of the approach [14].

\section{MAPPINGS FOR HUMAN-ROBOT TEAM INTERACTION}

The control paradigm introduced in Sec. II can be used for human-robot team interaction. In particular, in this paper we focus on a bilateral telemanipulation scenario where the hand of a human operator is put in correspondence with a robot team to perform a cooperative manipulation task. More specifically, we assume that the human provides control inputs for the object manipulation and grasp maintenance subtasks. We assume that it is possible to track the human hand motion and that each manipulator establishes a single contact point with the object. We further consider that it is possible to measure contact forces, $\boldsymbol{h}^{m}$. Following the definition introduced in [15] and further developed in [16], we define as forward mapping the procedure necessary to obtain at the slave side (robot team) the desired motions, $\boldsymbol{v}_{c}^{d}$ and $\boldsymbol{v}_{r}^{d}$, captured on the master (human operator) side. The backward mapping computes the forces to be displayed to the human user, starting from the measurements acquired at the slave side, $\boldsymbol{h}_{c}^{m}$ and $\boldsymbol{h}_{r}^{m}$. In this work master and slave sides refer to the human operator's hand instrumented with wearable haptics and the robot team performing the task, respectively. This mapping is suitable for the single human-robot team interaction since it does not depend on the number of robots in the team as well as on their kinematics. Moreover, this approach allows the development of the interaction on the levels of subtasks, while the interaction with individual robots is not allowed. Therefore, within the mapping framework, forward mapping projects human commands onto the spaces of subtasks. Backward mapping projects the measured forces, generated as a result of the interaction with the object, to the space in which wearable fingertip haptic devices operate.

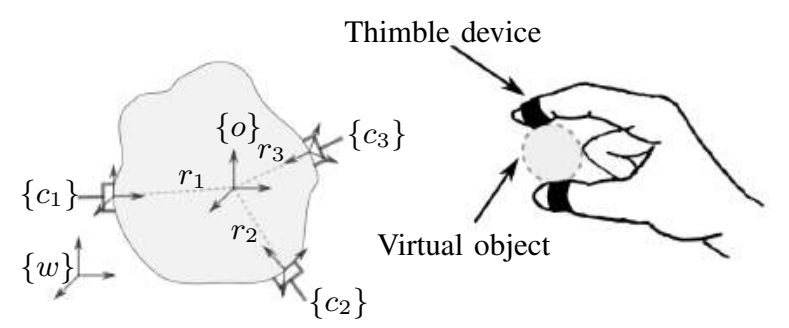

Fig. 2. Human-robot team interaction for cooperative manipulation. The human hand and finger motions are desired motions for the controlled cooperative and relative subtasks of the system. The motions are determined by the assumption that the human grasps and manipulates a virtual object. Human fingertips which are in "contact" with the virtual object are equipped with wearable fingertip haptic devices.

\section{A. Forward mapping}

In human-robot team interaction it is necessary to appropriately resolve assymmetry between the single human and the multiple robots. Therefore, we propose a mapping solution that transforms a command provided by human into appropriate desired commands for the robot team.

Let us assume the human operator is grasping a virtual object with $M$ contact points, $\boldsymbol{x}_{i}^{h}, i=1, \ldots, M$, as shown in Fig. 2. The virtual object is assumed to be a minimum volume sphere encircling all the contact points. Its pose is denoted as $\boldsymbol{x}_{v o}$. Let us assume the human performs grasp maintenance and object manipulation of the virtual object. Similar to the approach represented in Sec. II it is possible to compute the Jacobian matrices of the human's cooperative and relative behaviors, $\boldsymbol{J}_{c}^{h}$ and $\boldsymbol{J}_{r}^{h}$, respectively. The kinematic subtask decoupling approach for this case is sufficient as the dynamical behavior of the human fingers is neglected. Projection of the human fingers' motion onto the space of the virtual object motion, i.e. onto the cooperative subtask is

$$
\boldsymbol{v}_{c}^{h}=\boldsymbol{v}_{v o}=\boldsymbol{J}_{c}^{h}\left(\boldsymbol{x}^{h}\right) \boldsymbol{v}^{h},
$$

where $\boldsymbol{J}_{c}^{h}=G^{h^{\dagger T}}$ with $\boldsymbol{G}^{h}$ being the grasp matrix of the virtual human grasp, $\boldsymbol{v}^{h}$ is a stacked vector of the human contact points' velocities $\boldsymbol{v}^{h}=\left[\boldsymbol{v}_{1}^{h T}, \ldots, \boldsymbol{v}_{M}^{h T}\right]^{T}, \boldsymbol{v}_{v o}$ and $\boldsymbol{v}_{c}^{h}$ are the velocity vectors of the virtual object and cooperative behavior of the human, respectively.

The relative motion of the contact points defines the relative behavior of the human

$$
\boldsymbol{v}_{r}^{h}=\boldsymbol{J}_{r}^{h}\left(\boldsymbol{x}^{h}\right) \boldsymbol{v}^{h}
$$

where $\boldsymbol{J}_{r}^{h}$ represents the Jacobian of the relative subtask performed by the human. The relative velocity of the 


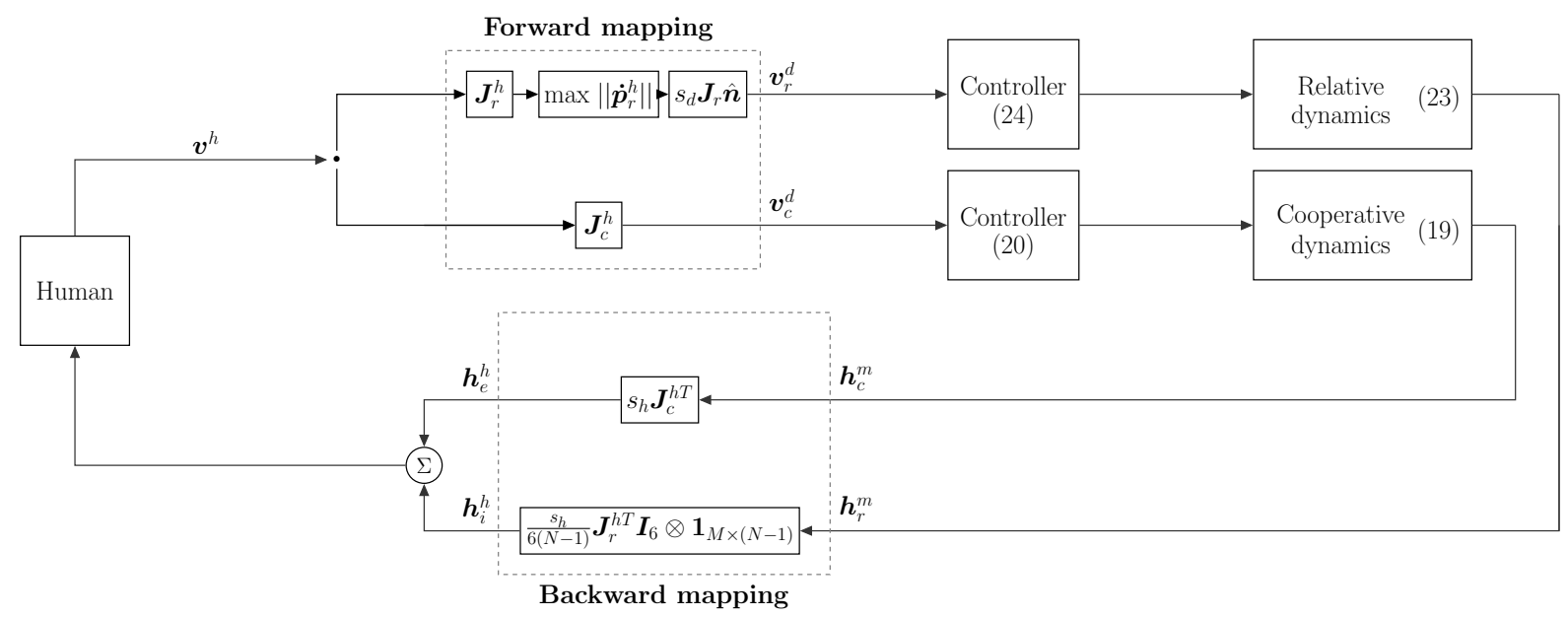

Fig. 3. Block structure of the control loop for human-robot team interaction.

human fingers is given with the stacked vector $\boldsymbol{v}_{r}^{h}=$ $\left[\dot{\boldsymbol{p}}_{r, 1}^{h T}, \mathbf{0}_{3}, \ldots, \dot{\boldsymbol{p}}_{r,(M-1)}^{h T}, \mathbf{0}_{3}\right]^{T}$. Relative behavior can be understood as the defornation of the elastic, virtual object. The consequence of this assumption is the variability of the virtual object radius

$$
\dot{r}_{v o}=\max \left\{\left\|\dot{\boldsymbol{p}}_{r, 1}^{h}\right\|, \ldots,\left\|\dot{\boldsymbol{p}}_{r,(M-1)}^{h}\right\|\right\} .
$$

Cooperative behavior of the human is mapped onto the cooperative subtask space of the robot team so that the human cooperative behavior is the desired control input for the cooperative controller of the robot team:

$$
\boldsymbol{v}_{c}^{d}=\boldsymbol{v}_{c}^{h} .
$$

The relative behavior of the robot team can be obtained by mapping the change of the virtual object radius to the space of the relative subtask of the robot team system:

$$
\boldsymbol{v}_{r}^{d}=s_{d} \dot{r}_{v o} J_{r} \hat{\boldsymbol{n}}
$$

where $s_{d}=\frac{r_{o}}{r_{v o}}$ is the scaling factor which takes into consideration different dimensions of the actual and virtual objects. The stacked vector of directions of normals in $\{w\}$ to the object surface for all end-effectors is provided with the vector $\hat{\boldsymbol{n}}$.

\section{B. Backward mapping}

Let us assume the human is equipped with $M$ wearable haptic fingertip devices as shown in Fig. 2. The wrenches measured by the robot team, $\boldsymbol{h}^{m}$, are fed-back to the human onto the space in which the fingertip devices operate.

The definition of a virtual object on the human side allows us to assume the following relation: the total wrench acting on the object grasped by the robot team is also acting on the virtual object defined on the human side, possibly scaled. Mapping the total forces onto the human side we obtain:

$$
\boldsymbol{h}^{h}=\boldsymbol{h}_{e}^{h}+\boldsymbol{h}_{i}^{h}=s_{h}\left(\boldsymbol{J}_{c}^{h T} \boldsymbol{h}_{c}^{m}+\boldsymbol{N}_{\boldsymbol{G}^{h}} \boldsymbol{\xi}\right)
$$

where $s_{h}=\frac{h_{\max }^{h}}{h_{\max }^{m}}$ represents the scaling factor that is the ratio between the maximum wrench that can be rendered by the $M$ haptic devices and the maximum wrench that is expected for the task. External wrench sensed by the human is $\boldsymbol{h}_{e}^{h}$ and the internal wrench sensed by the human is $\boldsymbol{h}_{i}^{h}$ with $\mathcal{N}_{G^{h}}$ being the null-space projector of the grasp matrix and $\boldsymbol{\xi} \in \mathbb{R}^{M}$ a vector parametrizing the homogeneous part of the solution.

The tuning of the homogeneous part in (31) is not unique in general and it is necessary to determine in which direction it is more convenient to render the forces. In following, we will consider a simplified case when two contact points are established on the master and the slave side. The computation of the relative forces that need to be rendered on the human side is

$$
\boldsymbol{h}_{i}^{h}=\frac{1}{6(N-1)} s_{h} \boldsymbol{J}_{r}^{h T} \boldsymbol{I}_{6} \otimes \mathbf{1}_{(M) \times(N-1)} \boldsymbol{h}_{r}^{m},
$$

where $I$ is an identity matrix, while $\mathbf{1}$ is the matrix of ones. If more contact points are taken in account, a possible solution to compute internal force directions is reported in [16]. A block diagram of the whole control loop for human-robot team telemanipulation is reported in Fig. 3.

\section{EXPERIMENTAL RESULTS}

In this section the performance of the proposed humanmulti robot interaction approach is experimentally evaluated under different conditions.

\section{A. Experimental Setup}

The setup used for the experimental validation consists of two 7 degrees of freedom KUKA LWR 4+ robotic manipulators. However, the approach is not limited by the number of robots used and can be easily extended to more manipulators, as seen in Fig. 4. The redundant joints are controlled by a stiffness oriented approach to keep the joint at a fixed configuration. The impedance parameters for the cooperative behavior are set to $\boldsymbol{D}_{c}=\operatorname{diag}\left(\left[\begin{array}{llllll}200 & 200 & 200 & 5 & 5 & 5\end{array}\right]\right)$, $k_{c}=500, \kappa_{c}=15$ and the true robot inertia for $\boldsymbol{M}_{c}$. For the relative behavior the corresponding parameters are chosen as $\boldsymbol{D}_{r}=\operatorname{diag}\left(\left[\begin{array}{llllll}300 & 300 & 300 & 5 & 5 & 5\end{array}\right]\right)$ and $k_{r}=500$. The 


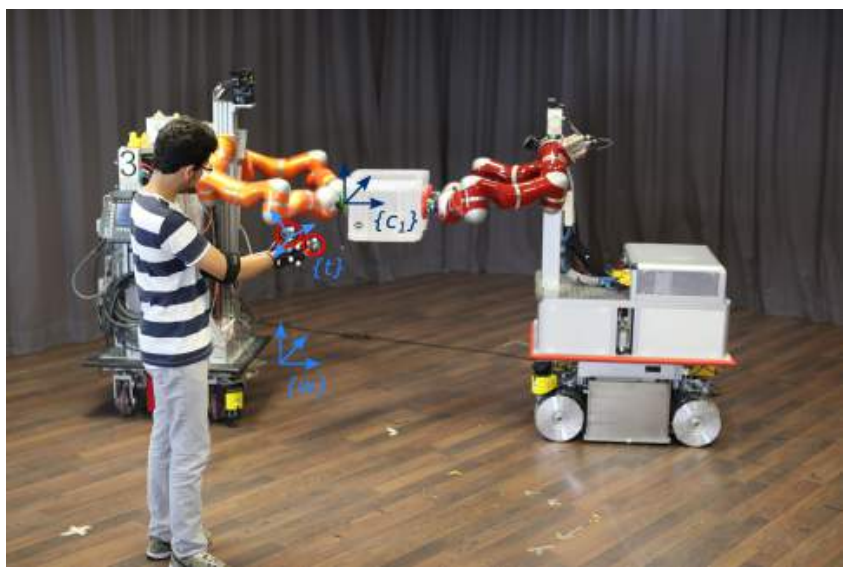

Fig. 4. Experimental setup with four KUKA LWR 4+ robotic manipulators and a human expert, equipped with wearable thimble haptic devices (marked with red circles). The end-effector $\{r\}$, wearable thimble $\{t\}$ and world frames $\{w\}$ are drawn in blue.

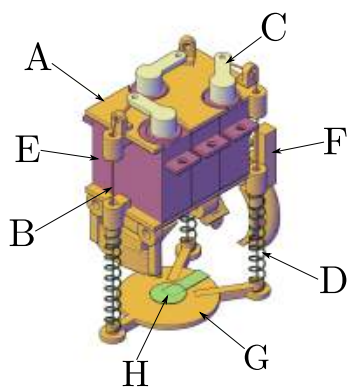

Fig. 5. The actuated thimble device. A rigid body (A) houses three servomotors (E) connected through three wires (B) to the vertices of a slanting surface $(\mathrm{G})$. The surface is located under the finger pulp of the user whose distal phalanx finger holds the thimble in its position using a clamp (F). A force sensor $(\mathrm{H})$ located between the finger pulp and the slanting surface is employed to set the calibration of the platform relatively to the finger size. The initial position of the platform is held by three springs (D). When motors are not activated the orientation of the platform is parallel to the body (A).

external wrenches acting on each end-effector are estimated internally by the KUKA from measured torques in the joint space and known Jacobian. The desired virtual object motion as well as the relative motion is obtained by tracking the human with the marker-based motion tracking system Qualisys. Furthermore, the human operator is equipped with two wearable thimble devices, which are fitted on the thumb and index finger, respectively. The actuated thimble devices and its elements are depicted in Fig. 5. Three motors control the length of the three tensors which are connected to the vertices of the slanting surface (letter $G$ in Fig. 5). The tensors pull independently the three vertices on the platform resulting in the 3 degrees of freedom: roll $\alpha$, pitch $\beta$ and displacement $d$. The internal forces are mapped as: $\alpha=$ $\operatorname{atan}\left(\frac{{ }^{t} f_{r, x}^{m}}{{ }^{t} f_{r, z}^{m}}\right), \beta=\operatorname{atan}\left(\frac{{ }^{t} f_{r, y}^{m}}{{ }^{t} f_{r, z}^{m}}\right)$ and $d=s_{h} k_{t} \sqrt{{ }^{t} \boldsymbol{f}_{r}^{m T}{ }^{m} \boldsymbol{f}_{r}^{m}}$, with the scaling factor $s_{h}=\frac{4.7}{30}$ and the fingertip compliance parameter $k_{t}=2 \mathrm{~mm} / \mathrm{N}$ [17]. The transformation of the frame of the wearable thimble devices $t_{j}, j=1, . ., M$ (located at point $(\mathrm{H})$ in Fig. 5) relative to the frame of the end-effectors $c_{i}, i=1, \ldots, N$ is assumed to be known. The
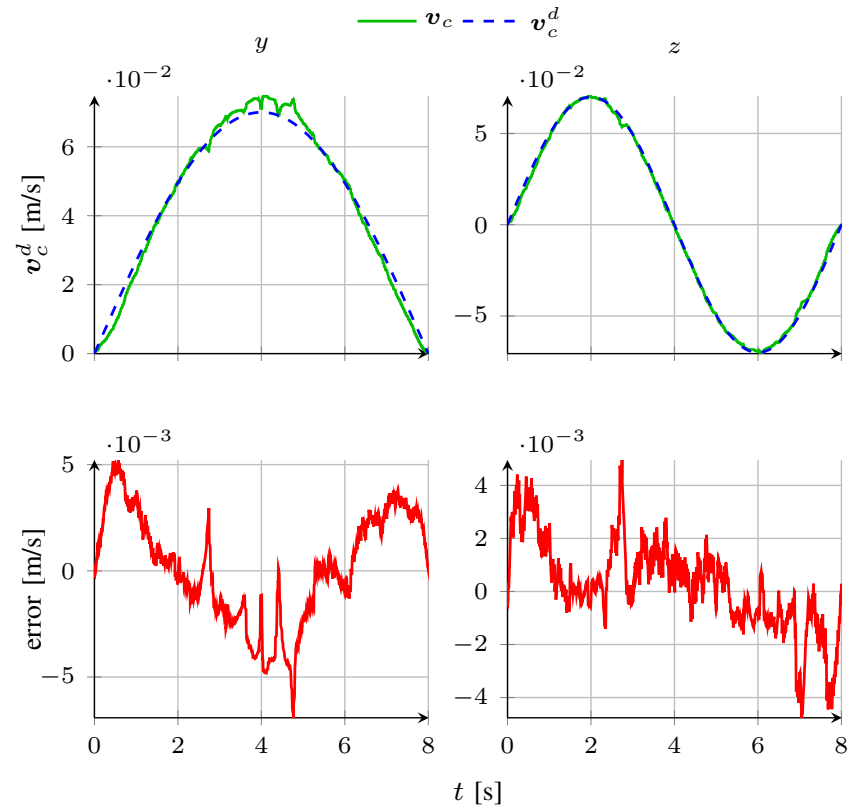

Fig. 6. Velocity (top) and tracking error (bottom) of desired (blue) and actual (green) $y, z$ components (left to right), without human operator. The error spikes result from object dynamics.

devices are equipped with a FSR 400 sensor from Interlink Electronics to measure forces fed back to the human operator. Similar devices as well as details on the principle of work are presented in [8], [18], [19]. The devices are connected to a Raspberry Pi which ensures wireless communication with the robot system. The sampling frequency is $1 \mathrm{kHz}$ for the controller and $0.1 \mathrm{kHz}$ for the Qualisys motion tracking system as well as the thimble devices. The evaluation of the proposed approach is split into four experiments:

(i) Translational motion of cooperative behavior, without human-in-the-loop, by applying $\boldsymbol{v}_{c}^{d}=\boldsymbol{a} \sin (2 \pi \boldsymbol{f} t)$, with the amplitude $\boldsymbol{a}=[0,0.07,0.07]^{T} \mathrm{~m} / \mathrm{s}$ and frequency $\boldsymbol{f}=[0,1 / 16,1 / 8]^{T} \mathrm{~Hz}$, resulting in a typical motion profile of a pick and place task in the $y-z$ plane.

(ii) Angular motion tracking of cooperative behavior, without human-in-the-loop, by applying angular velocities $\omega_{r, z}^{d}=a \sin (2 \pi f t)$, along the $z$-axis, with the amplitude $a=-0.07 \mathrm{rad} / \mathrm{s}$ and frequency $f=1 / 6 \mathrm{~Hz}$.

(iii) Position tracking of relative behavior, without humanin-the-loop, by applying $\boldsymbol{v}_{r, y}^{d}= \pm 0.05 \mathrm{~m} / \mathrm{s}$, in $y$-axis, for each end-effector, during the approach phase. After an impact force of $30 \mathrm{~N}$ is detected the manipulation phase starts and a fixed set point distance is commanded to maintain the grasp.

(iv) Pick and place task, with human-in-the-loop commanding velocities and receiving feedback from the two wearable thimble devices.

\section{B. Results}

Experiment (i): Fig. 6 depicts the desired and resulting actual velocity for the cooperative subtask as well as the resulting error, for the commanded directions in the $y-z$ 


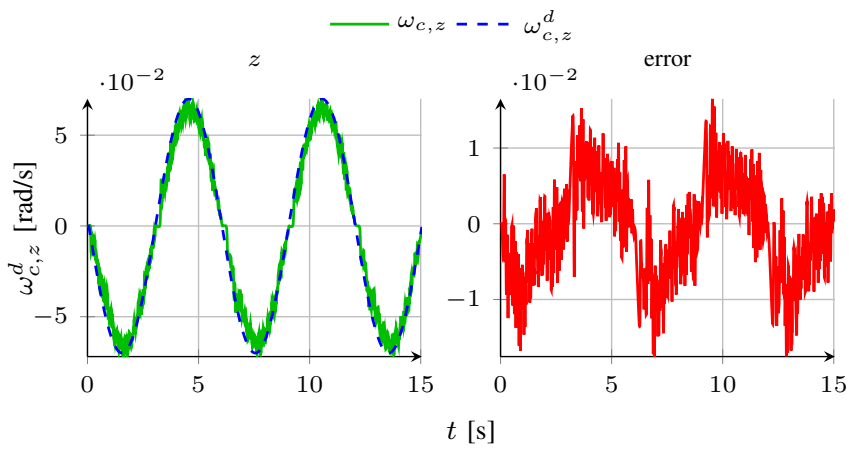

Fig. 7. Angular velocity (left) and tracking error (right) of desired (blue) and actual (green) $z$ component, without human operator.

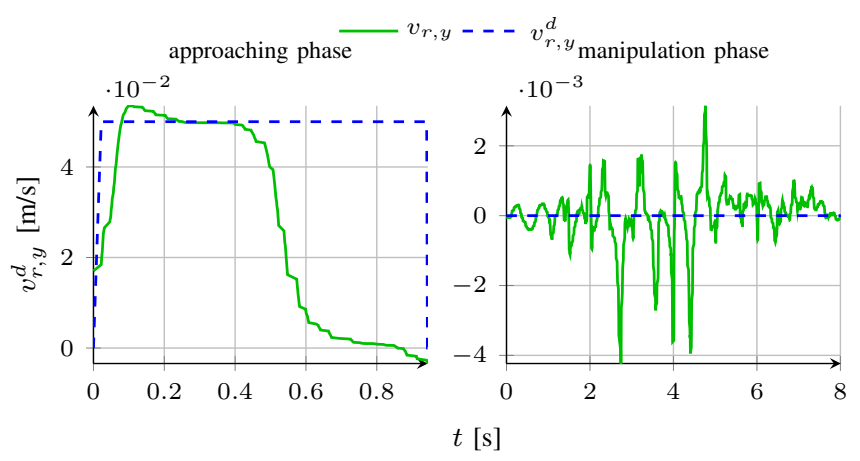

Fig. 8. Velocity of desired (blue) and actual (green) $y$ components, during approach (left) and manipulation (right) phase and without human operator. When contact is established the relative velocity approaches zero due to hard world constraints. The small error in the manipulation phase conforms decoupling of the cooperative and relative subtasks.

plane. Good tracking can be observed, with a mean error of $\left[\begin{array}{ll}2.3 & 8.0\end{array}\right]^{T} \times 10^{-5} \mathrm{~m} / \mathrm{s}$, for $y-$ and $z-$ axes, respectively. The spike in error of $[5.24 .4]^{T} \times 10^{-3} \mathrm{~m} / \mathrm{s}$ at the start and $\left[\begin{array}{ll}3.8 & -4.8\end{array}\right]^{T} \times 10^{-3} \mathrm{~m} / \mathrm{s}$ at the end of the motion is due to the influence of the object dynamics on the impedance controller.

Experiment (ii): The desired and resulting actual angular velocity for the cooperative subtask as well as the resulting error are presented in Fig. 7. It can be observed that good tracking of angular velocities is achieved with a mean error of $-8.0481 \times 10^{-4} \mathrm{rad} / \mathrm{s}$ in $z$-axis. Similar to Experiment (i) the error spikes of up to \pm 0.017 result from object dynamics.

Experiment (iii): The commanded and actual relative velocity, the error of relative dynamics for the approaching and the manipulation phase are depicted in Fig. 8. After an initial overshoot producing an error of $3.2 \times 10^{-2} \mathrm{~m} / \mathrm{s}$ in $y$-axis, the constant velocity is achieved until the impact with the object, where the error grows up to $5.3 \times 10^{-2} \mathrm{~m} / \mathrm{s}$ due to hard world constraints. The resulting energy stored in the virtual stiffness of the impedance controller maintains a rigid grasp. During the manipulation phase the error between desired and actual relative velocity averages at $2.6 \times 10^{-3} \mathrm{~m} / \mathrm{s}$ confirming the dynamic decoupling of the relative subtask from the cooperative subtask.

Experiment (iv): The resulting motion profile of a hu-

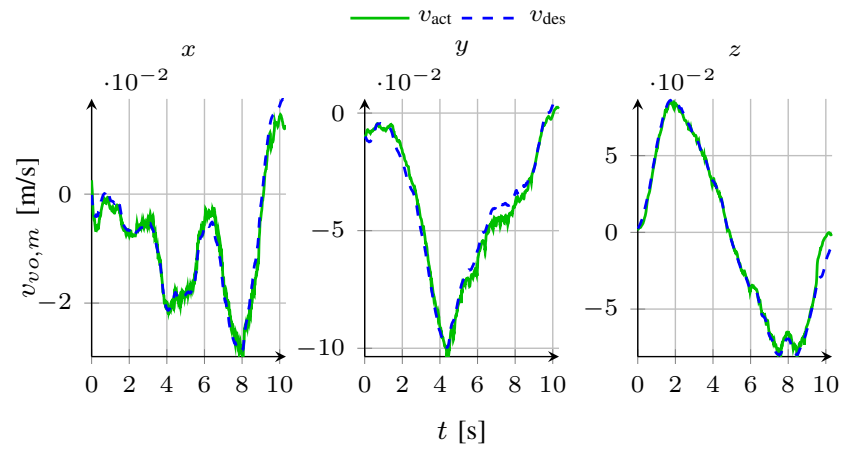

Fig. 9. Velocity of desired (blue) and actual (green) $x, y, z$ components (left to right), with object, commanded by a human.

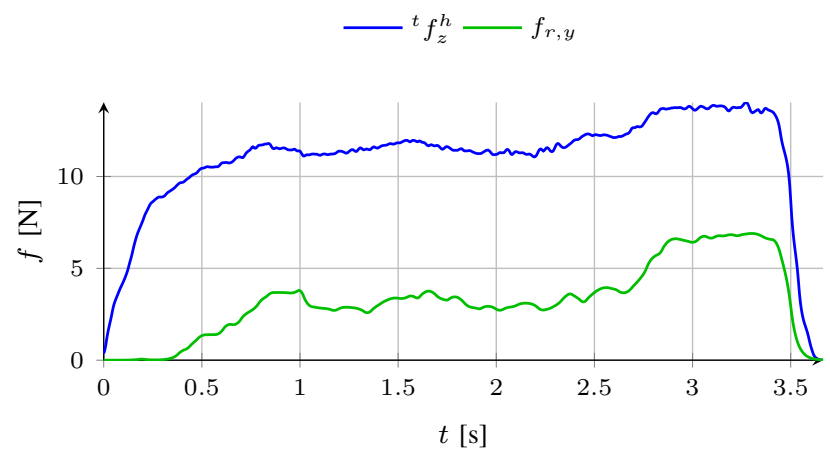

Fig. 10. Internal force at the robot (blue) compared to feedback provided to the human operator (green) along the $y$-axis. A stable grasp is maintained and reasonable transparency is achieved. The force feedback is rescaled for visibility, as only the profile of the force is of importance.

man expert fulfilling a pick and place task is presented in Fig. 9. With errors in the interval of $[-4,5.3] \times 10^{-3}$, $[-6.7,10.2] \times 10^{-3},[-5.6,5.7] \times 10^{-3} \mathrm{~m} / \mathrm{s}$, for $x-, y-$ and $z-$ axes, it can be seen that desired human velocities, which are typically more abrupt in their profile than artificially generated motions, are successfully tracked. Fig 10 depicts the profile of internal forces $f_{r, y}$ acting on the object along the $y$-axis as well as the internal force feedback ${ }^{t} f_{z}^{h}$, provided to the human expert, along the corresponding axis of the thimble device $z$. The force feedback being zeros at the start and the end of the motion can be explained by the sensing techniques used. Due to the curvature of the finger as well as it not being aligned with the force sensor perfectly, small forces cannot be measured well. However, as the general force profiles are compared, meaningful results can still be obtained. It can be seen that ${ }^{t} f_{z}^{h}$ follows $f_{r, y}$ and the human operator is able to keep the internal force nearly constant at an average of $12.1 \mathrm{~N}$ during object manipulation, thus indicating reasonable transparency of the system. The influence of different types of feedback on the human is currently being investigated.

In this section promising results for the proposed human multi robot interaction approach were presented, for linear as well as for angular motions. Furthermore, the dynamic decoupling of cooperative and relative behavior is shown as well as the applicability in a realistic pick and place task. In 
the future we plan to further extend the control approach to be able to track a desired relative force applied to the object.

\section{CONCLUSION}

In this paper a novel human-robot team interaction setting in a teleoperation mode for a cooperative manipulation task is proposed. We propose the dynamical subtask-based control approach to perform multiple subtasks. Within the control architecture two subtasks are considered: cooperative (object manipulation) and relative (grasp maintenace). Both subtasks are commanded by the human via suitable forward mapping. The feedback of the current state of the robot team is provided via wearable haptic thimble devices through suitable backward mapping. Experimental results validate the implemented control architecture. We show that novel forms of interaction are possible with wearable haptic devices which ensure the mobility of the human during the interaction with a robot system.

In future we plan to extend the control approach to multiple subtasks and to evaluate the human performance in the proposed setting.

\section{ACKNOWLEDGMENTS}

The work is supported by the European Union Seventh Framework Programme FP7/2007-2013 under grant agreement no. 601165 of the project "WEARHAP - Wearable Haptics for Humans and Robots".

\section{REFERENCES}

[1] S. Musić and S. Hirche, "Classification of human-robot team interaction paradigms," in 1st IFAC Conference on Cyber-Physical \& HumanSystems, 2016, 2016.

[2] D. Lee and M. W. Spong, "Bilateral teleoperation of multiple cooperative robots over delayed communication networks: theory," in Proceedings of the 2005 IEEE International Conference on Robotics and Automation. IEEE, 2005, pp. 360-365.

[3] A. Franchi, C. Masone, V. Grabe, M. Ryll, H. H. Bülthoff, and P. R. Giordano, "Modeling and control of uav bearing formations with bilateral high-level steering," The International Journal of Robotics Research, vol. 31, no. 12, pp. 1504-1525, 2012.

[4] G. Gioioso, A. Franchi, G. Salvietti, S. Scheggi, and D. Prattichizzo, "The flying hand: a formation of uavs for cooperative telemanipulation," in IEEE International Conference on Robotics and Automation (ICRA), 2014.
[5] D. Sieber, S. Music, and S. Hirche, "Multi-robot manipulation controlled by a human with haptic feedback," in IEEE/RSJ International Conference on Intelligent Robots and Systems (IROS), 2015.

[6] T. Hatanaka, N. Chopra, and M. Fujita, "Passivity-based bilateral human-swarm-interactions for cooperative robotic networks and human passivity analysis," in 2015 54th IEEE Conference on Decision and Control (CDC). IEEE, 2015, pp. 1033-1039.

[7] Z. Wang and M. Schwager, "Force-amplifying n-robot transport system (force-ants) for cooperative planar manipulation without communication," The International Journal of Robotics Research, vol. 35 no. 13, pp. 1564-1586, 2016.

[8] F. Chinello, M. Malvezzi, C. Pacchierotti, and D. Prattichizzo, "Design and development of a 3rrs wearable fingertip cutaneous device," in IEEE/ASME International Conference on Advanced Intelligent Mechatronics, 2015.

[9] G. Antonelli, F. Arrichiello, and S. Chiaverini, "The null-space-based behavioral control for autonomous robotic systems," Intelligent Service Robotics, vol. 1, no. 1, pp. 27-39, 2008. [Online]. Available: http://dx.doi.org/10.1007/s11370-007-0002-3

[10] A. Dietrich, C. Ott, and A. Albu-Schäffer, "An overview of null space projections for redundant, torque-controlled robots," The International Journal of Robotics Research, vol. 34, no. 11, pp. 1385-1400, 2015.

[11] C. Ott, A. Dietrich, and A. Albu-Schäffer, "Prioritized multi-task compliance control of redundant manipulators," Automatica, vol. 53, pp. 416-423, 2015.

[12] D. Prattichizzo and J. Trinkle, "Grasping," in Handbook on Robotics, B. Siciliano and O. Kathib, Eds. Springer, 2008, pp. 671-700.

[13] F. Caccavale, P. Chiacchio, A. Marino, and L. Villani, "Sixdof impedance control of dual-arm cooperative manipulators,' IEEE/ASME Transactions on Mechatronics, 2008.

[14] C. Ott, A. Kugi, and Y. Nakamura, "Resolving the problem of non-integrability of nullspace velocities for compliance control of redundant manipulators by using semi-definite lyapunov functions," in 2008 IEEE International Conference on Robotics and Automation, May 2008, pp. 1999-2004.

[15] G. Salvietti, L. Meli, G. Gioioso, M. Malvezzi, and D. Prattichizzo, "Object-based bilateral telemanipulation between dissimilar kinematic structures," in IEEE/RSJ International Conference on Intelligent Robots and Systems (IROS), 2013.

[16] _ - "Multicontact bilateral telemanipulation with kinematic asymmetries," IEEE/ASME Transactions on Mechatronics, vol. 22, no. 1, pp. 445-456, Feb 2017.

[17] K.-H. Park, B.-H. Kim, and S. Hirai, "Development of a soft-fingertip and its modeling based on force distribution," in Robotics and Automation, 2003. Proceedings. ICRA'03. IEEE International Conference on, vol. 3. IEEE, 2003, pp. 3169-3174.

[18] D. Prattichizzo, F. Chinello, C. Pacchierotti, and M. Malvezzi, "Towards wearability in fingertip haptics: a 3-dof wearable device for cutaneous force feedback," IEEE Transactions on Haptics, 2013.

[19] C. Pacchierotti, L. Meli, F. Chinello, M. Malvezzi, and D. Prattichizzo, "Cutaneous haptic feedback to ensure the stability of robotic teleoperation systems," International Journal of Robotics Research, vol. 34, no. 14, pp. 1773-1787, 2015. 